\title{
Biologia e tabela de vida do ácaro-vermelho Tetranychus bastosi em pinhão-manso
}

\author{
Marçal Pedro Neto(1), Renato Almeida Sarmento(1), Wennder Parente de Oliveira(1), Marcelo Coutinho Picanço(2) \\ e Eduardo Andrea Lemus Erasmo(1)
}

\begin{abstract}
(1)Universidade Federal do Tocantins, Campus de Gurupi, Caixa Postal 66, CEP 77402-970 Gurupi, TO. E-mail: pedronetom@yahoo.com.br, rsarmento@uft.edu.br, wpo@uft.edu.br, erasmolemus@uft.edu.br (2)Universidade Federal de Viçosa, Departamento de Entomologia, Avenida P.H. Rolfs, s/no, CEP 36571000 Viçosa, MG. E-mail: picanco@ufv.br
\end{abstract}

\begin{abstract}
Resumo - O objetivo deste trabalho foi determinar a biologia e a tabela de vida de Tetranychus bastosi em pinhão-manso (Jatropha curcas). O experimento foi realizado em ambiente controlado a $26^{\circ} \mathrm{C}$ e $75 \%$ de UR, com fotófase de 12 horas. Os ovos usados nos experimentos foram oriundos de criação estoque. As avaliações foram realizadas duas vezes ao dia, para a biologia do ácaro, e uma vez, para os parâmetros reprodutivos. O ciclo médio de vida das fêmeas foi de 9,63 dias e o dos machos, de 8,94 dias. A razão sexual foi 0,65 e a longevidade média das fêmeas foi de 16 dias, com produção média de 59 ovos por fêmea. Os parâmetros de tabela de vida obtidos foram: taxa líquida de reprodução $\left(R_{o}\right), 45,41$ indivíduos; duração média das gerações (T), 12,66 dias; taxa intrínseca de crescimento $\left(\mathrm{r}_{\mathrm{m}}\right), 0,0538$ fêmea por fêmea por dia; razão finita de aumento $(\lambda)$, 1,023 fêmea por fêmea; e tempo para duplicação da população (TD), 3,15 dias. O ácaro T. bastosi desenvolvese bem e apresenta alto potencial reprodutivo sobre folhas de pinhão-manso.
\end{abstract}

Termos para indexação: Jatropha curcas, potencial reprodutivo, Tetranychidae.

\section{Biology and life table of the red spider mite Tetranychus bastosi on physic nut}

\begin{abstract}
The objective of this work was to determine the biology and life table of Tetranychus bastosi on physic nut (Jatropha curcas). The experiment was carried out under controlled conditions at $26^{\circ} \mathrm{C}$ and $75 \% \mathrm{RH}$, with photophase of 12 hours. The eggs used in the experiments were obtained from stock culture. Evaluations were performed twice a day for the biology of the mite, and once a day for reproductive parameters. The average life cycle of females was 9.63 days and that of males was 8.94 days. The sex ratio was 0.65 , and the mean longevity of females was 16 days, with average production of 59 eggs per female. The life table parameters obtained were: liquid reproduction rate $\left(\mathrm{R}_{\mathrm{o}}\right), 45.41$ individuals; average generation length $(T), 12.66$ days; intrinsic growth rate $\left(\mathrm{r}_{\mathrm{m}}\right), 0.0538$ female per female per day; finite growth rate $(\lambda), 1.023$ female per female; and time required for doubling the population (TD), 3.15 days. The spider mite T. bastosi develops well and presents a high reproductive potential on leaves of physic nut.
\end{abstract}

Index terms: Jatropha curcas, reproductive potential, Tetranychidae.

\section{Introdução}

Entre as plantas com potencial para produção de biodiesel, o pinhão-manso (Jatropha curcas L.; Euphorbiaceae) destaca-se pela elevada produção e qualidade do óleo extraído das suas sementes (Saturnino et al., 2005; Severino et al., 2007; Andrade et al., 2008). Contudo, essa cultura é atacada por diversos artrópodes-praga, como o ácaro-vermelho Tetranychus bastosi Tuttle, Baker \& Sales, 1977 (Acari: Tetranychidae), documentado como um dos mais danosos (Saturnino et al., 2005; Santos et al., 2010; Sarmento et al., 2011; Cruz et al., 2012).
A principal característica dos ácaros da família Tetranychidae é a produção de teia, cuja principal função é a proteção dos ovos contra a dessecação e o forrageamento por predadores (Sabelis \& Bakker, 1992; Venzon et al., 2009; Franco et al., 2010). Esses ácaros são classificados como polífagos e cosmopolitas. Além disso, apresentam alto potencial reprodutivo, a depender da planta hospedeira e das condições climáticas (Silva et al., 2009; Cruz et al., 2013). O gênero Tetranychus Dufour, 1832, é o principal dessa família de ácaros, na qual se encontram cerca de $50 \%$ dos ácaros fitófagos conhecidos no mundo (Jeppson et al., 1975). 
A oviposição de T. bastosi inicia-se após as fêmeas produzirem teia sobre as folhas da planta hospedeira. Em condições favoráveis, a folha é rapidamente tomada pela teia produzida por esse ácaro, o que diminui sua capacidade fotossintética e prejudica o crescimento das plantas (Santos et al., 2010).

Tetranychus bastosi é relatado no Nordeste, no Sudeste e no Norte do Brasil, associado a plantas de crescimento natural e a plantas do gênero Jatropha (Tuttle et al., 1977). Para o uso adequado das estratégias de controle da praga, é importante o conhecimento de seus aspectos biológicos e reprodutivos.

O objetivo deste trabalho foi determinar a biologia e a tabela de vida de T. bastosi em pinhão-manso.

\section{Material e Métodos}

$\mathrm{O}$ experimento foi conduzido em sala climatizada a $26 \pm 1^{\circ} \mathrm{C}$ e $70 \pm 5 \%$ de UR, com fotófase de 12 horas. Os ácaros usados foram retirados de criação estoque e criados em folha de pinhão-manso circundada por camada de algodão hidrófilo fixada sobre espuma de plástico umedecida com água destilada, em uma bandeja de 26x37 cm (Polanczyk et al., 2011). As folhas foram obtidas aleatoriamente de plantas cultivadas em casa de vegetação.

Foram avaliados ciclo biológico, viabilidade dos ovos, sobrevivência dos estádios de desenvolvimento dos ácaros e razão sexual de T. bastosi. Para tanto, foram confeccionadas três arenas, com $3 \mathrm{~cm}$ de diâmetro, em cada folha. Para a limitação da área de cada arena, foi utilizada cola entomológica para impedir a fuga dos ácaros. As folhas foram circundadas por tiras de algodão hidrofóbico e acondicionadas em placa de Petri (15 cm de diâmetro), sob espuma de plástico umedecida para garantir maior viabilidade à folha. Cada arena recebeu duas fêmeas adultas acasaladas de T. bastosi, que permaneceram nas arenas por 16 horas, para oviposição. Em seguida, as fêmeas foram retiradas das arenas e os ovos quantificados, tendo-se deixado apenas um ovo por arena.

Para o período de incubação e a fase de desenvolvimento, foram realizadas duas avaliações diárias com estereomicroscópio (às 8 e às 16 h), visto que alguns estádios duram menos de 24 horas. As repetições consistiram das arenas contendo um ovo, num total de 120 parcelas experimentais. A razão sexual foi determinada após a quantificação do número de fêmeas e machos emergidos, oriundos das posturas das fêmeas fertilizadas da criação estoque. A viabilidade dos ovos foi calculada pela diferença entre o número de ovos deixados nas arenas e o número de ovos não eclodidos. O erro-padrão das médias foi calculado com o programa estatístico R (R Development Core Team, 2011).

Para confecção da tabela de vida, os aspectos reprodutivos de $T$. bastosi foram analisados nos períodos de pré-oviposição, oviposição e pós-oviposição, tendo-se determinado o número de ovos diários e a longevidade de fêmeas e machos. A sexagem foi feita após os adultos emergirem, pela observação de características morfológicas, como coloração, formato e tamanho do corpo, opistossoma do macho e idiossoma da fêmea (Jeppson et al., 1975; Moraes \& Flechtmann, 2008). Nas arenas apenas com fêmeas, foram colocados machos, e, nas que continham apenas machos, foi avaliada a longevidade. Os ovos depositados por fêmea foram quantificados e eliminados a cada 24 horas.

Os dados de sobrevivência (lx), fertilidade específica $(\mathrm{mx})$, taxa líquida de reprodução $\left(\mathrm{R}_{\mathrm{o}}\right)$, duração média de uma geração $(T)$, razão finita de aumento $(\lambda)$ e tempo necessário para a população duplicar o números de indivíduos (TD) foram observados em casais formados por fêmeas obtidas de ovos usados nas avaliações do ciclo biológico e um ácaro macho, nas arenas para a confecção da tabela de vida (Silveira Neto et al., 1976). Os casais foram observados diariamente, tendo-se quantificado e retirado os ovos depositados no período. Os machos mortos foram substituídos, para restabelecer o casal. Caso a fêmea morresse, a repetição era finalizada. Foram observadas 61 fêmeas de $T$. bastosi, cada uma considerada como repetição.

\section{Resultados e Discussão}

A duração média total do desenvolvimento de fêmeas de T. bastosi, de ovo a adulto, foi de 9,63 dias (com mínimo de oito e máximo de 11 dias). Para os machos, a duração foi de 8,94 dias em média (com mínimo de oito e máximo de dez dias) (Tabela 1).

O período de incubação médio foi de 4,18 dias para machos e fêmeas, enquanto o desenvolvimento do período móvel das fêmeas (larva, protoninfa e deutoninfa) apresentou média de 1,17 e 1,10 dia, respectivamente. Em relação ao período imóvel pós-embrionário (protocrisalida, deutocrisalida e teliocrisalida), as médias obtidas foram de 0,67, 0,68 
e 0,97 dia, respectivamente. Os machos apresentaram período de desenvolvimento móvel (larva, protoninfa e deutoninfa) com média de 0,98, 0,69 e 0,93 dia, respectivamente. Já os períodos imóveis pós-embrionários (protocrisalida, deutocrisalida e teliocrisalida) apresentaram média de 0,66, 0,66 e 0,87 dia, respectivamente (Tabela 1 ).

Em trabalhos realizados com o parente próximo T. urticae Koch, 1836 , a $25^{\circ} \mathrm{C}$, em folhas de gérbera, o ciclo de ovo a adulto foi, em média, de 13,8 dias (Silva et al., 2009). Para a espécie T. desertorum Banks, 1900, o período de desenvolvimento em feijoeiro foi de 6,80 dias (Rivero \& Vásquez, 2009). Silva (2002) relatou ciclo de desenvolvimento de 13,29 dias para T. ludeni Zacher, em algodoeiro. O ciclo biológico é inerente à espécie, mas também é influenciado pela temperatura e pelo hospedeiro, entre outros fatores. No Estado de Tocantins, a temperatura média anual é de $26^{\circ} \mathrm{C}$, o que o torna um ambiente favorável para o desenvolvimento de T. bastosi, por possibilitar menor ciclo biológico. Isso contribui para explicar as altas infestações ocorridas em lavouras de pinhão-manso no estado, no início do período chuvoso (Cruz et al., 2013).

A viabilidade de ovos foi de $94 \%$, com sobrevivência de $98 \%$, para larvas, e de $91 \%$ para o período de ovo-adulto. A razão sexual foi de $0,65 \%$ (Tabela 2 ). Com relação aos aspectos reprodutivos, foi observado período de pré-oviposição, oviposição e pós-oviposição de 1,39, 15 e 1,16 dias, respectivamente, com produção média de 59 ovos por fêmea (Tabela 3). Os machos alcançaram longevidade de 23 dias, superior à das fêmeas, o que pode ser explicado pelo maior gasto

Tabela 1. Duração em dias (média \pm erro-padrão) de fases de desenvolvimento de Tetranychus bastosi em pinhão-manso (Jatropha curcas).

\begin{tabular}{|c|c|c|c|c|}
\hline \multirow[t]{2}{*}{ Fases } & \multicolumn{2}{|c|}{ Fêmea } & \multicolumn{2}{|c|}{ Macho } \\
\hline & $\mathrm{N}^{(1)}$ & Média & $\mathrm{N}$ & Média \\
\hline Ovo & 66 & $4,18 \pm 0,06$ & 36 & $4,13 \pm 0,06$ \\
\hline Larva & 66 & $1,17 \pm 0,04$ & 36 & $0,98 \pm 0,03$ \\
\hline Protocrisalida & 66 & $0,67 \pm 0,03$ & 36 & $0,66 \pm 0,04$ \\
\hline Protoninfa & 66 & $0,82 \pm 0,04$ & 36 & $0,69 \pm 0,06$ \\
\hline Deutocrisalida & 66 & $0,68 \pm 0,03$ & 36 & $0,66 \pm 0,05$ \\
\hline Deutoninfa & 66 & $1,10 \pm 0,04$ & 36 & $0,93 \pm 0,06$ \\
\hline Teliocrisalida & 66 & $0,97 \pm 0,03$ & 36 & $0,87 \pm 0,04$ \\
\hline Ovo-adulto & 66 & $9,63 \pm 0,09$ & 36 & $8,94 \pm 0,01$ \\
\hline Longevidade & 60 & $16,00 \pm 1,14$ & 26 & $25,00 \pm 2,03$ \\
\hline
\end{tabular}

${ }^{(1)} \mathrm{N}$, número de observações. energético destas com a produção de ovos (Oku et al., 2009).

Foi observado maior número de ovos no segundo dia de oviposição, com média de dez ovos por dia. Esse valor diminuiu acentuadamente até o quinto dia. Após

Tabela 2. Parâmetros populacionais de Tetranychus bastosi em folha de pinhão-manso (Jatropha curcas).

\begin{tabular}{lc}
\hline Parâmetro & Valor \\
\hline Taxa intrínseca de crescimento (fêmeas por fêmea por dia) & 0,22 \\
Razão finita de aumento (indivíduos por fêmea) & 1,25 \\
Taxa líquida de reprodução (fêmeas por fêmea) & 45,41 \\
Duração média de uma geração (dias) & 17,17 \\
Tempo para dobrar a população (dias) & 3,15 \\
Razão sexual & 0,65 \\
\hline
\end{tabular}

Tabela 3. Tabela de vida de fertilidade de Tetranychus bastosi em pinhão-manso (Jatropha curcas).

\begin{tabular}{|c|c|c|c|c|}
\hline $\begin{array}{l}\text { Intervalo } \\
\text { de idade } \\
(\mathrm{X}) \\
\end{array}$ & $\begin{array}{l}\text { Fertilidade } \\
\text { específica } \\
(\mathrm{MX})\end{array}$ & $\begin{array}{c}\text { Probabilidade } \\
\text { de sobrevivência } \\
\text { (LX) }\end{array}$ & $\begin{array}{c}\mathrm{MX} \times \\
\mathrm{LX}\end{array}$ & $\begin{array}{l}\mathrm{MX} \times \\
\mathrm{LX} \times \\
\mathrm{X}\end{array}$ \\
\hline 11,5 & 2,58 & 1,00 & 2,58 & 29,69 \\
\hline 12,5 & 4,31 & 1,00 & 4,31 & 53,83 \\
\hline 13,5 & 6,20 & 1,00 & 6,20 & 83,74 \\
\hline 14,5 & 5,79 & 1,00 & 5,79 & 83,91 \\
\hline 15,5 & 5,38 & 1,00 & 5,38 & 83,44 \\
\hline 16,5 & 4,14 & 1,00 & 4,14 & 68,23 \\
\hline 17,5 & 2,97 & 0,98 & 2,91 & 50,98 \\
\hline 18,5 & 2,69 & 0,95 & 2,55 & 47,26 \\
\hline 19,5 & 1,95 & 0,85 & 1,66 & 32,35 \\
\hline 20,5 & 2,35 & 0,75 & 1,76 & 36,17 \\
\hline 21,5 & 2,22 & 0,62 & 1,38 & 29,58 \\
\hline 22,5 & 1,88 & 0,57 & 1,07 & 24,06 \\
\hline 23,5 & 2,01 & 0,46 & 0,92 & 21,70 \\
\hline 24,5 & 1,80 & 0,39 & 0,70 & 17,23 \\
\hline 25,5 & 1,76 & 0,36 & 0,64 & 16,19 \\
\hline 26,5 & 1,90 & 0,32 & 0,61 & 16,14 \\
\hline 27,5 & 1,62 & 0,28 & 0,45 & 12,51 \\
\hline 28,5 & 1,99 & 0,25 & 0,50 & 14,18 \\
\hline 29,5 & 0,90 & 0,25 & 0,22 & 6,60 \\
\hline 30,5 & 1,74 & 0,25 & 0,44 & 13,28 \\
\hline 31,5 & 1,19 & 0,25 & 0,30 & 9,40 \\
\hline 32,5 & 1,00 & 0,25 & 0,25 & 8,08 \\
\hline 33,5 & 0,93 & 0,20 & 0,19 & 6,25 \\
\hline 34,5 & 0,90 & 0,16 & 0,14 & 4,94 \\
\hline 35,5 & 1,03 & 0,13 & 0,13 & 4,74 \\
\hline 36,5 & 0,85 & 0,11 & 0,09 & 3,42 \\
\hline 37,5 & 0,60 & 0,08 & 0,05 & 1,79 \\
\hline 38,5 & 0,50 & 0,05 & 0,02 & 0,96 \\
\hline 39,5 & 0,25 & 0,03 & 0,01 & 0,29 \\
\hline 40,5 & 0,37 & 0,02 & 0,01 & 0,30 \\
\hline Somatório & - & - & 45,51 & 781,24 \\
\hline
\end{tabular}


o oitavo dia, constatou-se nova redução na oviposição diária (Figura 1).

Espécies do gênero Tetranychus têm mostrado valores de oviposição próximos aos observados no presente trabalho. $\mathrm{O}$ pico de oviposição de $T$. desertorum, em feijoeiro, foi alcançado no quarto dia, com 6,93 ovos por dia (Rivero \& Vásquez, 2009). Silva et al. (2009), em estudo com fêmeas de T. urticae em gérbera, relataram valores médios de 3,75 ovos por fêmea por dia, próximos aos encontrados no presente trabalho.

De acordo com Oku et al. (2009), espécies da família Tetranychidae produzem menor quantidade de teia quando têm maior disponibilidade de recursos para aumentar outras aptidões, como número de ovos produzidos. Portanto, a alta taxa de oviposição obtida para T. bastosi no presente trabalho - quando comparada à de outras espécies do mesmo gênero -, além de ser uma característica inerente à espécie e a sua adaptação ecológica à disponibilidade de recursos no ambiente, pode consistir, também, numa estratégia de compensação à maior vulnerabilidade a predadores decorrente da menor quantidade de teia produzida.

A longevidade de 16 dias das fêmeas de T. bastosi (Tabela 1) foi superior aos dez dias registrados para T. desertorum (Rivero \& Vásquez, 2009). A sobrevivência $\left(1_{x}\right)$ de $T$. bastosi foi de $95 \%$ até o oitavo dia de oviposição, com rápida queda a partir do nono dia (Figura 1). No décimo terceiro dia, a mortalidade de fêmeas foi superior a 50\%.

Os parâmetros da tabela de vida obtidos para estimar a capacidade de crescimento da população do ácaro-praga $T$. bastosi foram: taxa líquida de reprodução $\left(\mathrm{R}_{\mathrm{o}}=45,41\right.$ fêmeas por fêmea); duração

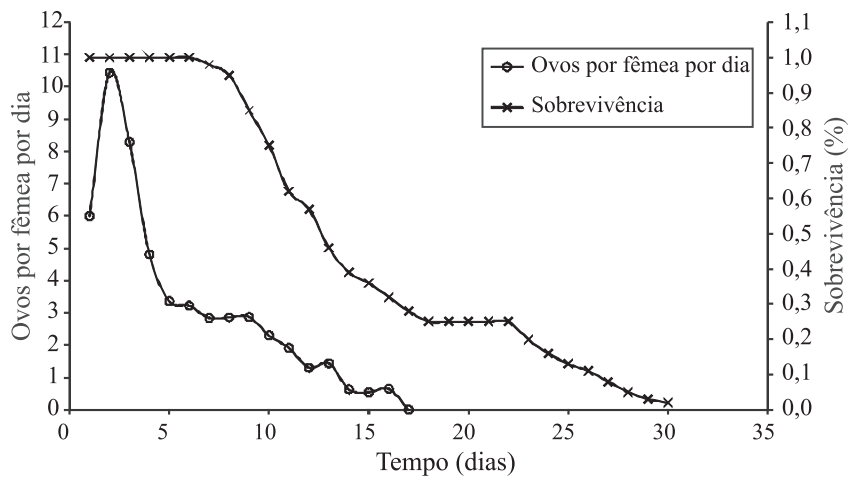

Figura 1. Reprodução e sobrevivência de Tetranychus bastosi em pinhão-manso (Jatropha curcas). média de uma geração ( $\mathrm{T}=17,17$ dias); razão finita de aumento ( $\lambda=1,25$ indivíduo por fêmea); taxa intrínseca de crescimento $\left(\mathrm{r}_{\mathrm{m}}=0,22\right.$ fêmea por fêmea por dia); e tempo necessário para a população duplicar o número de indivíduos ( $\mathrm{TD}=3,15$ dias) (Tabelas $2 \mathrm{e}$ 3). Esses resultados são próximos aos encontrados para T. ludeni, em folhas de algodoeiro (Silva, 2002). A taxa intrínseca de crescimento de T. bastosi assemelhou-se a valores comumente encontrados para espécies da família Tetranychidae. Por exemplo, para T. mexicanus Banks, 1900, a taxa intrínseca de crescimento foi de 0,24, em Annona muricata, e de 0,21 em A. coriaceae (Sousa et al., 2010). Quanto à média de uma geração (T), Silva et al. (2009) relataram valor semelhante para a $T$. urticae $(18,6)$, em gérbera, e Sousa et al. (2010), para $T$. mexicanus $(18,66)$, em $A$. muricata, enquanto Rivero \& Vásquez (2009) obtiveram valores superiores para $T$. desertorum $(11,45)$ em feijoeiro. A taxa de reprodução $\left(R_{0}\right)$ foi próxima à encontrada para T. desertorum $(41,10)$ em feijoeiro (Rivero \& Vásquez, 2009), inferior à encontrada para $T$. mexicanus $(85,45)$ (Sousa et al., 2010) e superior à observada para $T$. urticae $(11,5)$ em gérbera (Silva et al., 2009). A razão finita de aumento $(\lambda)$ foi próxima à encontrada para as espécies $T$. desertorum e T. mexicanus.

\section{Conclusões}

1. O ácaro-praga Tetranychus bastosi desenvolvese bem e apresenta alto potencial reprodutivo sobre folhas de pinhão-manso.

2. O período médio de desenvolvimento é de 9,63 dias, para as fêmeas, e de 8,94 dias para os machos.

\section{Referências}

ANDRADE, G.A.; CARAMORI, P.H.; SOUZA, F.S. de; MARUR, C.J.; RIBEIRO, A.M.A. de. Temperatura mínima letal para plantas jovens de pinhão-manso. Bragantia, v.67, p.799-803, 2008. DOI: 10.1590/S0006-87052008000300031.

CRUZ, W.P. da; SARMENTO, R.A.; TEODORO, A.V.; ERASMO, E.A.L.; PEDRO NETO, M.; IGNACIO, M.; FERREIRA JUNIOR, D.F. Acarofauna em cultivo de pinhão-manso e plantas espontâneas associadas. Pesquisa Agropecuária Brasileira, v.47, p.319-327, 2012. DOI: 10.1590/S0100-204X2012000300002.

CRUZ, W.P.; SARMENTO, R.A.; TEODORO, A.V.; P. NETO, M.; IGNACIO, M. Driving factors of the communities of phytophagous and predatory mites in a physic nut plantation and spontaneous plants associated. Experimental and Applied Acarology, 2013. DOI: $10.1007 / \mathrm{s} 10493-013-9663-0$. 
FRANCO, R.A.; REIS, P.R.; ZACARIAS, M.S.; OLIVEIRA, D.C. Influência da teia de Oligonychus ilicis (McGregor) (Acari: Tetranychidae) sobre os fitoseídeos predadores associados. Neotropical Entomology, v.39, p.97-100, 2010. DOI: 10.1590/ S1519-566X2010000100013.

JEPPSON, L.R.; KEIFER, H.H.; BAKER, E.W. Mites injurious to economic plants. Berkeley: University of California, 1975. 614p.

MORAES, G.J. de; FLECHTMANN, C.H.W. Manual de acarologia: acarologia básica e ácaros de plantas cultivadas no Brasil. Ribeirão Preto: Holos, 2008. 208p.

OKU, K.; MAGALHÃES, S.; DICKE, M. The presence of webbing affects the oviposition rate of two-spotted spider mites, Tetranychus urticae (Acari: Tetranychidae). Experimental and Applied Acarology, v.49, p.167-172, 2009. DOI: 10.1007/ s10493-009-9252-4.

POLANCZYK, R.A.; CELESTINO, F.N.; FERREIRA, L.S.; MELO, D.F.; BESTETE, L.R.; FRANCO, C.R.; PRATISSOLI, D. Desenvolvimento de Oligonychus ilicis em Coffea canephora sob diferentes temperaturas. Bragantia, v.70, p.370-374, 2011. DOI: 10.1590/S0006-87052011000200017.

R DEVELOPMENT CORE TEAM. R: a language and environment for statistical computing. Vienna: R Foundation for Statistical Computing, 2011.

RIVERO, E.; VÁSQUEZ, C. Biologia e tabela de vida de Tetranychus desertorum (Acari: Tetranychidae) sobre folhas de feijão (Phaseolus vulgaris). Zoologia, v.26, p.38-42, 2009. DOI: 10.1590/S1984-46702009000100007.

SABELIS, M.W.; BAKKER, F.M. How predatory mites cope with the web of their tetranychid prey: a functional view on dorsal chaetotaxy in the Phytoseiidae. Experimental and Applied Acarology, v.16, p.203-225, 1992. DOI: 10.1007/BF01193804.

SANTOS, H.O.D.; SILVA-MANN, R.; BOARI, A.D.J. Tetranychus bastosi Tuttle, Baker \& Sales (Prostigmata: Tetranychidae) mites on Jatropha curcas (Lineus) in Sergipe State, Brazil. Comunicata Scientiae, v.1, p.153-155, 2010.

SARMENTO, R.A.; RODRIGUES, D.M.; FARAJI, F.; ERASMO, E.A.; LEMOS, F.; TEODORO, A.V.; KIKUCHI, W.T.;
SANTOS, G.R. dos; PALLINI, A. Suitability of the predatory mites Iphiseiodes zuluagai and Euseius concordis in controlling Polyphagotarsonemus latus and Tetranychus bastosi on Jatropha curcas plants in Brazil. Experimental and Applied Acarology, v.53, p.203-214, 2011. DOI: 10.1007/s10493-010-9396-2.

SATURNINO, H.; PACHECO, D.; KAKIDA, J.; TOMINAGA, N.; GONÇALVES, N. Cultura do pinhão-manso (Jatropha Curcas L.). Informe Agropecuário, v.26, p.44-78, 2005.

SEVERINO, L.S.; VALE, L.S. do; BELTRÃO, N.E. de M. A simple method for measurement of Jatropha curcas leaf area. Revista Brasileira de Oleaginosas e Fibrosas, v.11, p.9-14, 2007.

SILVA, C.A.D. da. Biologia e exigências térmicas doácaro-vermelho (Tetranychus ludeni Zacher) em folhas de algodoeiro. Pesquisa Agropecuária Brasileira, v.37, p.573-580, 2002. DOI: 10.1590/ S0100-204X2002000500001.

SILVA, E.A.; REIS, P.R.; CARVALHO, T.M.B.; ALTOÉ, B.F. Tetranychus urticae (Acari: Tetranychidae) on Gerbera jamesonii Bolus and Hook (Asteraceae). Brazilian Journal of Biology, v.69, p.1121-1125, 2009. DOI: 10.1590/S1519-69842009000500016.

SILVEIRA NETO, S.; NAKANO, O.; BARBIN, D.; VILLA-NOVA, N.A. Manual de ecologia dos insetos. São Paulo: Agronômica Ceres, 1976. 429p.

SOUSA, J.M. de; GONDIM JUNIOR, M.G.C.; LOFEGO, A.C. Biologia de Tetranychus mexicanus (McGregor) (Acari: Tetranychidae) em três espécies de Annonaceae. Neotropical Entomology, v.39, p.319-323, 2010. DOI: 10.1590/ S1519-566X2010000300002.

TUTTLE, D.M.; BAKER, E.W.; SALES, F.M. Spider mites (Tetranychidae: Acarina) of the state of Ceará, Brazil. International Journal of Acarology, v.3, p.1-9, 1977. DOI: 10.1080/01647957708683070.

VENZON, M.; LEMOS, F.; SARMENTO, R.A.; ROSADO, M.C.; PALLINI, A. Predação por coccinelídeos e crisopídeo influenciada pela teia de Tetranychus evansi. Pesquisa Agropecuária Brasileira, v.44, p.1086-1091, 2009. DOI: 10.1590/ S0100-204X2009000900003.

$\overline{\text { Recebido em } 21 \text { de outubro de } 2012 \text { e aprovado em } 15 \text { de março de } 2013}$ 